\title{
Gastos com DPOC e câncer de pulmão, brônquios e traqueia para o SUS relacionados à Cidade de Altamira (PA) entre os anos de 2017 e 2020
}

\author{
Expenses with COPD and lung cancer, bronches and trache to SUS related to Altamira (PA) City
}

between the years 2017 to 2020

Gastos con EPOC y cáncer de pulmón, bronquios y tráquea para el SUS relacionados con la

Ciudad de Altamira (PA) entre los años 2017 e 2020

Recebido: 03/04/2021 | Revisado: 09/04/2021 | Aceito: 14/04/2021 | Publicado: 27/04/2021

\author{
Jade de Moraes Bezerra \\ ORCID: https://orcid.org/0000-0002-3380-502X \\ Universidade Federal do Pará, Brasil \\ E-mail: jade.moraes97@hotmail.com \\ Ana Beatriz Costa da Silva \\ ORCID: https://orcid.org/0000-0002-8149-8802 \\ Universidade Federal do Pará, Brasil \\ E-mail: beatriz199813@hotmail.com \\ Fernando Cesar de Sousa Braga \\ ORCID: https://orcid.org/0000-0003-2594-0982 \\ Universidade Federal do Pará, Brasil \\ E-mail: fernando-cesar@outlook.com \\ Ilano Oliveira da Silva \\ ORCID: https://orcid.org/0000-0002-0478-6368 \\ Universidade Federal do Pará, Brasil \\ E-mail: ilanooliveiradasilva@gmail.com \\ Giselle Sousa Carmona \\ ORCID: https://orcid.org/0000-0001-9299-2999 \\ Universidade Federal do Pará, Brasil \\ E-mail: gih_carmona1@icloud.com \\ Francisco Bruno Teixeira \\ ORCID: https://orcid.org/0000-0001-8596-3727 \\ Universidade Federal do Pará, Brasil \\ E-mail: teixeira.f.bruno@gmail.com
}

\begin{abstract}
Resumo
O objetivo do presente trabalho é estimar gastos públicos diretos com DPOC e câncer de pulmão, brônquios e traqueia na cidade de Altamira (PA), na região amazônica brasileira, entre os anos de 2017 e 2020. Trata-se de um estudo transversal baseado em dados oficiais e secundários do Sistema de Informação Hospitalares do Sistema Único de Saúde (SIH/SUS), fornecidos pelo Departamento de Informática do Sistema Único de Saúde (DATASUS) referentes aos anos de 2017 a 2020 relativos à cidade de Altamira (PA). O gasto com atendimentos hospitalares de moradores do município Altamira (PA) relacionados à DPOC e ao câncer de pulmão, traqueia, brônquios em outras cidades foi de R\$ 129.056,28. O custo para o poder público em internações no município em decorrência de internações hospitalares motivadas por essas patologias foi de $\mathrm{R} \$ 148.753,7$. A morte precoce, o sofrimento emocional e os significativos custos financeiros evitáveis são efeitos dessas doenças. O diagnóstico precoce, práticas de educação em saúde e apoio assistencial contra o tabagismo são fundamentais para mitigar o impacto econômico dessas enfermidades para o sistema público de saúde brasileiro e para promover melhoria da qualidade de vida da população altamirense.

Palavras-chave: Doença pulmonar obstrutiva crônica; Economia da saúde; Neoplasias broncopulmonares; Região amazônica.
\end{abstract}

\begin{abstract}
The objective of this work was to estimate direct public spending on COPD and lung, bronchial and tracheal cancer in Altamira city, in the Brazilian Amazon region, between the years 2017 to 2020. This is a cross-sectional study based on official and secondary data from the Information System Hospitals of the Unified Health System (SIH/SUS), provided by the Informatics Department of the Unified Health System (DATASUS) from 2017 to 2020 for Altamira (PA). The spent on hospital care for residents of the municipality of Altamira (PA) related to COPD and lung, trachea, bronchial cancer in other cities was $\mathrm{R} \$ 129,056.28$. The cost to the government in hospitalizations in the municipality as a result of hospitalizations motivated by these pathologies was $\mathrm{R} \$ 148,753.7$. The early death, emotional suffering and significant avoidable financial costs are effects of these diseases. So, early diagnosis, health education practices and
\end{abstract}


health care support against smoking are fundamental to mitigate the economic impact of these diseases on the Brazilian public health system and to promote an improvement in the life quality of Altamira's population.

Keywords: Bronchopulmonary neoplasms; Amazon region; Chronic obstructive pulmonary disease; Health economics.

\section{Resumen}

El presente trabajo tiene como objetivo valorar gasto público directo con EPOC y cáncer de pulmón, bronquios y tráquea en la ciudad de Altamira (PA), en la región amazónica brasileña, entre los años 2017 a 2020 . Se trata de un estudio transversal basado en datos oficiales y secundarios del Sistema de Información Hospitalaria del Sistema Único de Salud (SIH/SUS), proporcionados por el Departamento de Informática del Sistema Único de Salud (DATASUS) relativos a los años 2017 a 2020 referidos a la ciudad de Altamira (PA). Resultados: El gasto con atención hospitalaria de residentes del municipio de Altamira (PA) relacionados a la EPOC y los cánceres de pulmón, bronquios y tráquea en otras ciudades ha sido R \$ 129.056,28. El costo para el poder público en internaciones en el municipio como resultado de hospitalizaciones motivadas por estas patologias ha sido de $\mathrm{R} \$ 148.753,7$. La muerte temprana, la angustia emocional y los costos financieros son evitables efectos de estas enfermedades. De esa manera, la detección precoz, prácticas de educación en salud, y apoyo asistencial contra el tabaquismo son fundamentales para mitigar el impacto económico de estas enfermedades para el sistema público de salud brasileño y para promover la mejora de la calidad de vida de la población altamirense.

Palabras clave: Economía de la salud; Enfermedad pulmonar obstructiva crónica; Neoplasia broncopulmonar; Región amazónica.

\section{Introdução}

O município de Altamira é a maior do Brasil e a segunda maior do planeta em extensão territorial, com aproximadamente 159.533,306 km² (IBGE,2020). O município é o mais populoso da mesorregião rica em Unidades de Conservação e comunidades indígenas do Sudoeste Paraense, que inclui também as cidades de Aveiro, Itaituba, Jacareacanga, Novo Progresso, Rurópolis, Trairão, Altamira, Anapú, Brasil Novo, Medicilândia, Pacajá, Senador José Porfírio, Uruará e Vitória do Xingu (Velásquez, Villas Boas \& Schwartzman, 2006; Silva et al.,2013). A população altamirense foi estimada no ano de 2020 em 115.969 pessoas (IBGE, 2020).

O PIB (Produto Interno Bruto) do município é de R \$ 2.722.306,91, porém 41,5\% da população possui rendimento mensal per capita de até 1/2 salário-mínimo (IBGE, 2018). Em 2018, a população ocupada era de 17,7\% e o salário médio mensal dos trabalhadores formais era em média de 2,4 salários-mínimos (IBGE, 2018). As principais atividades econômicas da região de Altamira são a exploração madeireira, pecuária, agricultura, pesca, extrativismo, mineração industrial, garimpo, comércio e serviços (Neto \& Herrera, 2016; Ministério do Meio Ambiente, 2013; Romão et al., 2017).

A Usina Hidrelétrica de Belo Monte, a maior unicamente brasileira, está localizada nas cidades de Altamira, Brasil Novo, Vitória do Xingu, Anapu e Senador José Porfírio, e teve suas obras iniciadas em 2013 e início de operações em 2016 (Ladislau et al., 2016; Fainguelernt, 2016; Freire, de Lima \& da Silva, 2018). O processo de construção dessa geradora de energia elétrica foi importante para o desenvolvimento socioeconômico dessa área, todavia causou e ainda provoca impactos expressivos para o meio ambiente e para a comunidade indígena local (Fainguelernt, 2020).

O município de Altamira integra a Região de Saúde Xingu, uma subdivisão realizada pela Secretaria Estadual de Saúde do Estado do Pará (SESPA) para auxiliar o gerenciamento financeiro e o fluxo de atendimento no estado, a qual inclui também as cidades de Anapu, Brasil novo, Medicilândia, Pacajá, Porto de Moz, Senador José Profirio, Uruará e Vitoria do Xingu (SESPA, 2016). Os encaminhamentos para outros municípios quando a oferta for insuficiente ou inexistente na cidade de origem é feita de acordo com a portaria No 1.097 de 22 de maio de 2006 que define o processo da Programação Pactuada e Integrada da Assistência em Saúde no âmbito do SUS (Brasil, 2006). O município de referência (Altamira), único da Região em Saúde Xingu a possuir serviços de média e alta complexidade como pneumologia, assume o compromisso de atender aos encaminhamentos pactuados entre os gestores para atendimento da população residente em outros centros urbanos e deve ser aprovado na respectiva Comissão Intergestores Bipartite - CIB.

Conforme a World Health Organization (WHO), em 2019, a Doença Pulmonar Obstrutiva Crônica (DPOC) foi a 
terceira maior causa de morte no mundo e a sétima maior causa de anos de vida perdidos seja por morte precoce ou por falta de saúde plena no mundo (WHO, 2020). A prevalência na América foi de 14,53\% e na Europa de 13,29\%, entretanto, mais de $12 \%$ da população mundial sofre com DPOC (Varmaghani et al., 2019).

No Brasil, estima-se que a prevalência da DPOC foi de $19 \%$ da população adulta acima de 40 anos, no ano de 2018 , e que 17\% dos brasileiros são vítimas dessa patologia no Brasil (Cruz \& Pereira, 2020). Em 2019, essa doença foi a sexta maior causa de óbitos no Brasil.

Há décadas se sabe que mais de $80 \%$ dos casos dessa enfermidade são provocados pelo tabagismo e que pacientes com DPOC tem risco 10 a 14 vezes maior de morrer caso sejam usuários de tabaco (Thun et al., 1982). No Brasil, dos óbitos causados pelo cigarro, cerca de, $74 \%$ são decorrentes de tal doença crônica e, aproximadamente, $78 \%$ derivam de câncer de pulmão (Pinto et al., 2017).

A DPOC consiste em doenças causadoras de obstrução persistente de vias respiratórias que resulta em prejuízo à fase expiratória da ventilação pulmonar (Jezler et al., 2007). A exposição do indivíduo a substâncias danosas às vias aéreas provoca inflamação que lesa estruturas do sistema respiratório, como brônquios, sendo denominada de bronquite crônica, e alvéolos, chamada então de enfisema pulmonar por causar a destruição do conteúdo alveolar de elastina (Bagatin, Jardim \& Stirbulov, 2006; Barnes, 2004). O diagnóstico para DPOC envolve a avaliação clínica de sinais, sintomas e fatores de risco associada ao exame de espirometria e o tratamento pode ser realizado com uso de fármacos, abandono do tabaco, fisioterapia e cirurgia, a depender da gravidade do quadro (Brasil, 2013).

Em 2018, os diagnósticos de câncer de pulmão representaram cerca de 11,6\% dos novos casos de neoplasias malignas no mundo (Ferlay et al., 2019). Tal tumor maligno atualmente é responsável por aproximadamente 12,9\% de todos os novos casos de câncer nos Estados Unidos, correspondendo a uma estimava de 538.243 pessoas vivendo com câncer de pulmão em 2016 nos EUA (Howlader et al., 2016). Na Europa, 497.283 pessoas possuíam câncer de pulmão no ano de 2018 (Ferlay et al., 2019). Os cânceres de pulmão, brônquios e traqueia são a segunda maior causa de morte dentre os tumores malignos entre as mulheres e a primeira entre os homens no Brasil (INCA, 2020).

O diagnóstico desses cânceres do aparelho respiratório envolve avaliação de sinais e sintomas, exames de imagem, como radiografia e tomografia computadorizada, e biópsia. Hochhegger et al., (2015) afirma que a quimioterapia, a radioterapia e a ressecção cirúrgica são os mais atuais recursos terapêuticos disponíveis contra o tumor maligno pulmonar. Desse modo, conforme a American Cancer Society (2010), tais neoplasias malignas custam aproximadamente US\$180 bilhões por ano às economias mundiais.

No Brasil, os custos com diagnóstico, tratamento e acompanhamento do paciente com DPOC e câncer de pulmão, brônquios e traqueia impõem despesas expressivas para o SUS (Sistema Único de Saúde) e, assim, acabam por desviar recursos para doenças inevitáveis (Pinto, Pichon-Riviere \& Bardach, 2015).

De acordo com Pesquisa Especial de Tabagismo (PETab) realizada pelo Instituto Nacional de Câncer (INCA), as medidas de combate ao tabagismo foram adotadas por muitos países, como o Brasil, e já se mostraram eficazes para prevenção e retardo da evolução de doenças (INCA, 2011). Todavia, tais políticas públicas precisam ser ainda mais ampliadas no território continental brasileiro, pois, são ferramentas de ótimo custo-benefício para a União.

O presente trabalho busca expor uma estimativa de gastos públicos com DPOC e câncer de pulmão, brônquios e traqueia na cidade de Altamira, no estado do Pará, com intuito de contribuir para o adequado planejamento de medidas de combate a tais doenças pelo município. 


\section{Metodologia}

Trata-se de um estudo transversal baseado em dados oficiais e secundários do Sistema de Informações Hospitalares do Sistema Único de Saúde (SIH/SUS), fornecidos pelo Departamento de Informática do Sistema Único de Saúde (DATASUS) referentes aos anos de 2017, 2018, 2019 e 2020 relativos à cidade de Altamira-PA. O trabalho optou por abordar a morbidade e os gastos diretos referentes à DPOC e à neoplasia maligna de pulmão, brônquios e traqueia. As informações desta pesquisa foram fornecidas pela seleção dos termos "município", "lista morb CID-10", "faixa etária 1", "sexo", "cor/raça" e "valor total" disponíveis na plataforma virtual DATASUS.

O Sistema de Informações Hospitalares (SIH) usado neste trabalho forneceu dados acerca dos hospitais associados ao SUS. O município de Altamira possui quatro hospitais, sendo três públicos - Hospital Geral de Altamira São Rafael, Hospital Público Geral de Castelo dos Sonhos e Hospital Regional Público da Transamazônica - e um privado conveniado ao SUS Hospital Santo Agostinho - os quais são denominados "locais de internação" neste estudo (DATASUS, 2021). Porém, o Hospital Público Geral de Castelo dos Sonhos foi inaugurado apenas no fim do ano de 2020, enquanto os demais estiveram em funcionamento durante todo o período do presente estudo.

O local de internação é onde está a unidade hospitalar, enquanto o local de residência se refere à localidade de moradia do paciente tratado.

As variáveis dependentes estudadas foram:

(a) Morbidade hospitalar por local de internação e por local de residência, sua distribuição por sexo, faixa etária e etnia no município de Altamira - PA;

(b) Custos públicos diretos das internações na cidade de Altamira - PA.

Por tratar-se de um trabalho baseado em informações fornecidas pelas fichas de Autorização de Internação Hospitalar (AIH), há limitações relevantes:

I. não é possível contabilizar recursos municipais e estaduais destinados ao tratamento hospitalar das doenças abordadas no estudo;

II. foram ignoradas as despesas de procedimentos diagnósticos e de alta complexidade associados ao tratamento das patologias referidas no trabalho;

III. não há informações suficientes para calcular o custo indireto, associado à redução da produção e da produtividade do trabalhador doente.

Não foi necessária a submissão a um Comitê de Ética em Pesquisa com Seres Humanos, pois o presente estudo utiliza dados secundários de livre acesso pelo sistema eletrônico de dados DATASUS.

\section{Resultados}

Ocorreu o registro de 40 internações hospitalares em Altamira devido à DPOC em 2017, 50 em 2018, 24 em 2019 e 17 em 2020 totalizando 131 (Tabela 1). Desse grupo, 64,88\% eram de pessoas do sexo masculino. A etnia mais comum entre os internados foi a parda, que pertencia a 91,60\% dos pacientes. A faixa etária mais afetada nesse grupo foi a de 60 a 69 anos.

Foram detectadas 42 internações por DPOC em 2017, 46 em 2018, 25 em 2019 e 23 em 2020 de moradores da cidade de Altamira fora do município, totalizando 136 (Tabela 1). Destes, 63,50\% eram homens. A etnia mais recebida nesses locais de atendimento foi a parda, constituindo $81,75 \%$ das hospitalizações. A faixa etária mais comprometida foi a de 60 a 69 anos.

Sendo assim, cerca de $\mathrm{R} \$ 248.127,76$ foram gastos com pacientes que moram ou que buscaram atendimento hospitalar em Altamira-PA relacionado à DPOC, sendo R \$ 144.455,96 por local de internação e os restantes R $\$ 103.671,8$ por local de residência (Tabela 2). 
A respeito das internações em hospitais de Altamira atribuídas aos cânceres de pulmão, brônquios e traqueia, ocorreram 2 em 2017, nenhuma em 2018, 1 em 2019 e 3 em 2020 (Tabela 1). Dentre essas hospitalizações, mais de 83,33\% eram de pessoas do sexo masculino. A etnia parda foi registrada em 66,66\% das internações. A idade mais frequente foi de 70 a 79 anos, representando $66,66 \%$ dos atendimentos.

Outrossim, foram registradas 4 internações devido à neoplasia maligna de pulmão, brônquios e traqueia de moradores de Altamira fora da cidade em 2017, 2 em 2018, 6 em 2019 e 6 em 2020 (Tabela 1). Destas, 55,5 \% de internações de pacientes do sexo feminino. A etnia mais comum entre os internados foi a parda, constituindo $72,22 \%$ dos doentes. A faixa etária mais comprometida foi a de 60 a 69 anos, representando 38,88\% dos casos.

Diante disso, foi estimado que $\mathrm{R} \$ 29.682,22$ foram pagos pelo poder público em decorrência de câncer de pulmão, brônquios e traqueia, sendo $\mathrm{R}$ \$ 25.384,48 referentes a internações de moradores do município altamirense fora da cidade e R $\$$ 4.297,74 relativos a atendimentos hospitalares em Altamira (Tabela 2).

De acordo com os dados da Tabela 1, o número de internações por local de residência de pacientes com DPOC teve um aumento de aproximadamente 9,5\% de 2017 para 2018, uma queda de quase 46\% de 2018 para 2019. Essa diminuição se manteve do ano de 2019 para 2020 com uma redução de 8\% no número de internações. Quanto ao local de internação, os casos de internações sofreram um aumento de $25 \%$ de 2017 até 2018 e, após isso, manteve-se em queda do período de 2018 até 2020.

Na tabela 1 também se nota uma oscilação quanto às internações por local de residência por câncer de pulmão, brônquios e traqueia, em que de 2017 para 2018 houve uma queda de 50\%, 2018 para 2019 um aumento significativo de 200\% e no ano subsequente esse valor se manteve. De 2017 a 2019, não houve registro de casos por local de internação para essas neoplasias. No entanto, no ano de 2019 até 2020 houve um aumento expressivo de $200 \%$.

Em relação aos custos relacionados ao local de residência, presentes na Tabela 2 para DPOC, houve um padrão de queda de 2017 para 2018 com quase $40 \%$ e $43 \%$ de 2018 até 2019. No ano subsequente, houve um aumento de $18,6 \%$ nesse valor. Na variável custo por local de internação, houve uma redução total de 57,8\% de 2017 a 2020.

Conforme a Tabela 2, houve uma queda total de 38,3\% dos custos relacionados ao local de residência do ano de 2017 a 2020 para essas neoplasias. De 2017 a 2019, não houve registro de casos por local de internação. Consequentemente, os custos de 2017 a 2019 são zero. No entanto, no ano de 2019 até 2020 houve um aumento expressivo de $200 \%$ na variável custo. Como não houve registros de casos por local de internação nos anos de 2017 e 2018, ocorreu um aumento significativo de $325 \%$ do ano de 2019 para 2020.

Tabela 1 - Nủmero de internações por doença pulmonar obstrutiva crônica (DPOC) e devido à neoplasia maligna de pulmão, brônquios e traqueia por local de residência e por local de internação em Altamira -PA.

\section{Número de internaçōes por doença pulmonar obstrutiva crônica (DPOC)}

Por local de residência Por local de internação
Número de internações devido à neoplasia maligna de pulmão, brônquios e traqueia

Por local de residência Por local de internação

\begin{tabular}{lllll}
\hline 2017 & 42 & 40 & 4 & 2 \\
2018 & 46 & 50 & 2 & 0 \\
2019 & 25 & 24 & 6 & 1 \\
2020 & 23 & 17 & 6 & 3 \\
\hline TOTAL & 136 & 131 & 18 & 6
\end{tabular}

Fonte: Elaborada pelos autores com base no Sistema de Internaçōes Hospitalares (SIH), 2020. 
Tabela 2 - Custos por doença pulmonar obstrutiva crônica (DPOC) e devido à neoplasia maligna de pulmão, brônquios e traqueia por local de residência e por local de internação em Altamira -PA.

Custos por doença pulmonar obstrutiva crônica (DPOC)

Por local de residência

\section{Por local de internação}

Custos devido à neoplasia maligna de pulmão, brônquios e traqueia
Por local de internação

\begin{tabular}{ccccc}
\hline 2017 & 44378,40 & 60976,36 & 7659,87 & 2284,77 \\
2018 & 26480,39 & 29266,67 & 3010,66 & 0 \\
2019 & 15005,0 & 28536,07 & 9992,52 & 383,44 \\
2020 & 17808,01 & 25676,86 & 4721,43 & 1629,53 \\
\hline TOTAL & 103671,8 & 144455,96 & 25384,48 & 4297,74
\end{tabular}

Fonte: Elaborada pelos autores com base no Sistema de Internações Hospitalares (SIH), 2020.

\section{Discussão}

O presente trabalho é o primeiro a abordar os custos de DPOC e de câncer de pulmão, traqueia e brônquios na cidade de Altamira, no estado do Pará, localizado na Amazônia Oriental. Tal artigo é relevante por oferecer levantamento de informações importantes para a gestão pública, a fim de auxiliar a União na elaboração ou alteração de medidas de prevenção e de profilaxia associadas às doenças supracitadas, conforme a realidade da comunidade local. Além disso, esta produção científica apoia a transparência em relação aos gastos governamentais em saúde, porquanto promove a propagação de conhecimento de interesse público para a comunidade, capaz de, desse modo, colaborar para a atenuação dos males sociais, emocionais e financeiros causados por tais enfermidades.

Nesse contexto, analisar a prevalência dos fatores de risco das patologias estudadas é de grande importância para direcionar esforços com o objetivo de reduzir as perdas financeiras do sistema de saúde estatal. Dessa forma, embora a predisposição genética seja decisiva para o desenvolvimento de neoplasias malignas relacionadas ao trato respiratório inferior e de DPOC, o tabagismo, a exposição a fatores ocupacionais, a idade avançada, o sexo e a etnia são condições de risco expressivas para essas doenças respiratórias (SBPT, 2004; Zamboni, 2002).

Em 1950, Richard Doll e Bradford Hill publicaram o descobrimento da relação entre câncer de pulmão e tabagismo (Doll \& Hill, 1950). Os autores E. Cuyler Hammond e Daniel Horn (1958) foram um dos pioneiros a relacionar, em 1958, disfunções cardiovasculares e especialmente patologias respiratórias e cânceres ao uso de tabaco. Desde então, o tabagismo é considerado um importante fator de risco para desenvolvimento de determinadas enfermidades, denominadas tabacorelacionadas (Burns, 2003; Forey, Thornton \& Lee, 2011; Gold, 2020).

Acerca dos fatores ocupacionais para doença pulmonar obstrutiva crônica, segundo a Sociedade Brasileira de Pneumologia (2004), a inalação de fumaça proveniente da queima da lenha e de pó e a condição socioeconômica podem contribuir decisivamente para o desenvolvimento dessa enfermidade. Outrossim, o câncer de pulmão pode se desenvolver também devido a situações associadas ao trabalho, a exemplo da exposição a hidrocarbonetos aromáticos policíclicos, piche, fuligem, éter bisclorometílico e éter metilclorometílico, cádmio e níquel (Algranti, Buschinelli \& De Capitani, 2010).

A mineração, a exploração de madeira e a extração de metais preciosos, importantes atividades econômicas da cidade de Altamira, são associadas ao aumento do risco para desenvolvimento de câncer de pulmão e de DPOC. Tais áreas de trabalho provocam a chamada "exposição ocupacional", que consiste na exposição de trabalhadores a substâncias maléficas à saúde, como o pó da madeira e o mercúrio, relacionado ao garimpo, resultando no desenvolvimento de patologias, a exemplo das tratadas neste trabalho (Veríssimo, Mendonça \& Meyer, 2013; Peng et al., 2020; Barcenas et al., 2005; Moran-Mendoza et al., 
2008; Bolund et al., 2018; Hancock et al., 2015; Girdler-Brown et al., 2008; Poinen-Rughooputh et al., 2016).

A pirâmide etária do município de Altamira, no estado do Pará, possui as bases mais largas em comparação ao topo, indicando, assim, que a população mais jovem é superior a idosa (Figura 1). Isso ocorre provavelmente em virtude da carência de políticas públicas que corroborem para a qualidade de vida e saúde da população, fato que reduz a longevidade dos cidadãos.

Figura 1 - Distribuição da população por sexo, segundo os grupos de idade Altamira (PA).

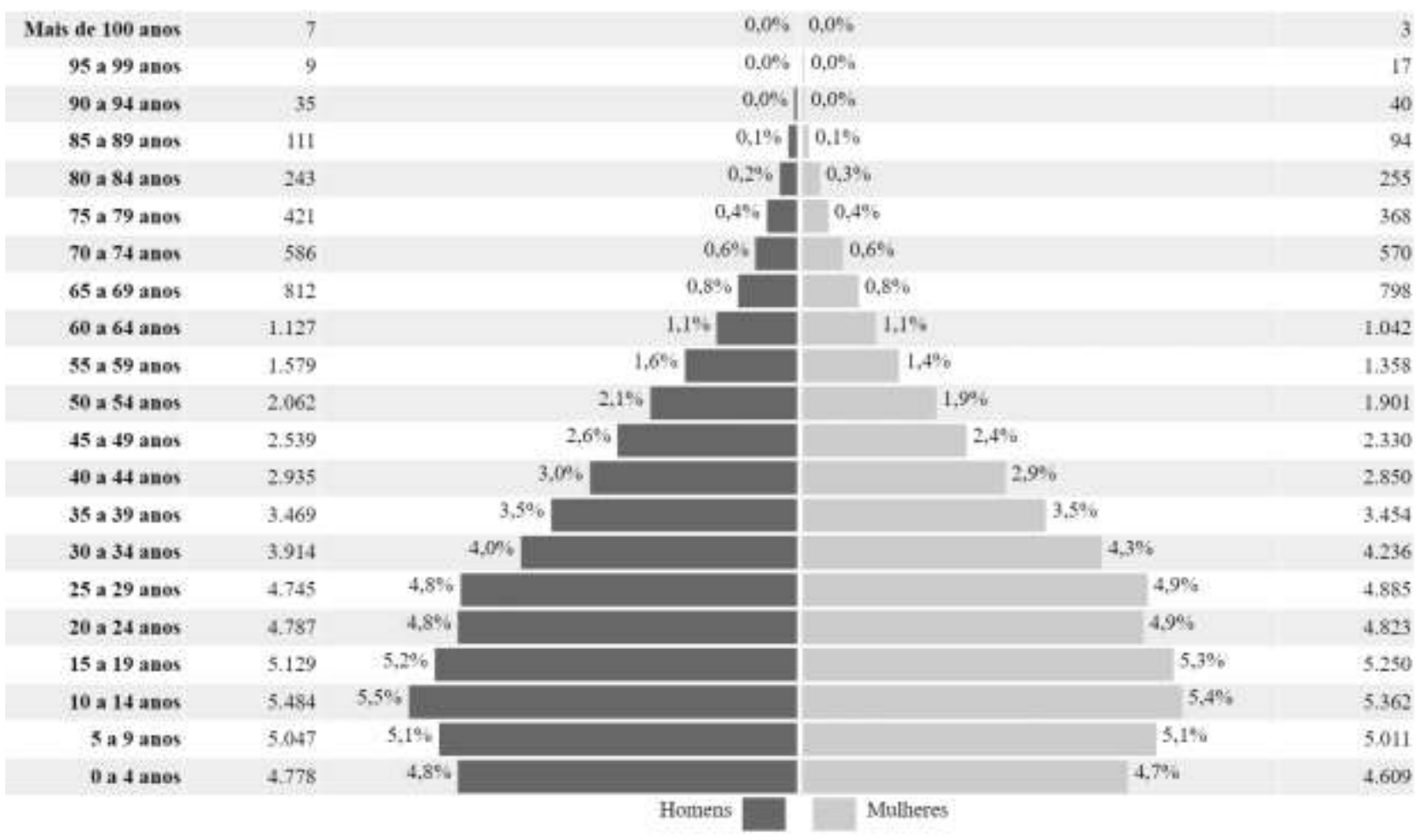

Fonte: Instituto Brasileiro de Geografia e Estatística (IBGE) (2020).

Soares et al., (2010) afirmam que a incidência da DPOC aumenta conforme a idade. Para Sales et al., (2018), pacientes com a doença são caracteristicamente mais velhos e com uma longa história de tabagismo. Os dados desta pesquisa foram concordantes com os estudos, com uma maior prevalência entre a sexta e a sétima década de vida entre os internados. Rabahi (2013) aponta como principais fatores de risco para a doença ter idade acima de 40 anos e histórico tabágico superior a 10 anos. Em relação a neoplasia maligna de pulmão, brônquios e traqueia, conforme os resultados alcançados, a faixa etária mais comprometida oscilou em pacientes de 60 a 79 anos de idade. Os dados foram condizentes com um estudo realizado por Carmo, Silva e Teixeira (2014) que obteve a maior frequência dessas neoplasias entre a sexta e a sétima década de vida.

Quanto à variável sexo, o presente estudo identificou que mais indivíduos biologicamente masculinos são internados em ambas as patologias. Para Torres et al., (2018), acredita-se que o fato de os homens serem os mais afetados esteja relacionado à história de consumo do tabaco, devido ao fato de o ato de fumar, no passado, ser considerado um comportamento associado à masculinidade. Tais autores apontam que esse cenário está em mudança e há, atualmente, um número expressivo de mulheres tabagistas que passaram a usar esse hábito como forma de ascensão e independência na sociedade.

Foi observada na pesquisa a maior ocorrência de DPOC em indivíduos pardos. Nota-se que este fenômeno está de acordo com constatações de Ejike et al., (2019), em que se destacou interações entre fatores de risco sociais, ambientais e genéticos comumente associados à DPOC precoce entre participantes pardos/negros. Tal explicação é válida também para os casos de câncer de pulmão, pois os negros têm maior chance de desenvolver esta doença (de Groot et al., 2018). Segundo 
Malta et al., (2015) a prevalência do tabagismo no Brasil é maior na população negra, havendo contraste com a população negra dos EUA, na qual a prevalência foi similar para brancos e negros. Apesar da prevalência do tabagismo ser similar em pessoas brancas e pardas/negras, as complicações de saúde são maiores entre os negros (Fagan et al., 2007). A presença do alelo CYP2A6*1B em pardos está associada a maior probabilidade do desenvolvimento do vício em nicotina (Balbani \& Montovani, 2005).

A partir disso, torna-se fundamental mensurar os efeitos financeiros resultantes da DPOC e câncer de pulmão, brônquios e traqueia a nível mundial e nacional, para correlacioná-los com os impactos monetários referentes à cidade de Altamira encontrados no estudo.

Apenas nos Estados Unidos da América (EUA), em 2010, foram projetados em aproximadamente US\$ 50 bilhões em perdas monetárias decorrentes de DPOC (Guarascio et al., 2013). Na Europa, os impactos econômicos resultantes dessa enfermidade também são significativos, representando o total de $€$ 48,4 bilhões em gastos para o ano de 2011 (ERS, 2013 ).

Em relação aos gastos nacionais com DPOC, segundo o DATASUS, atingiu-se R\$ 8.733.276,06 em 2017 , R\$ 105.477.181,88 em 2018, R\$ 107.114.901,81 em 2019 e R\$ 63.277.577,06 em 2020. Dessa forma, ao contrário da realidade brasileira na qual predominam aumentos anuais sucessivos nos custos de atendimento hospitalar de tais doenças, a cidade de Altamira apresenta predominante crescente queda nos gastos relativos a essas enfermidades. Os fatores que contribuíram para essa redução, provavelmente envolvem a redução no número de internações, as quais podem ser resultantes da finalização das obras de construção da usina hidrelétrica de Belo Monte, localizada na cidade paraense de Altamira, na Amazônia Oriental brasileira, somados às dificuldades de rastreio por parte da atenção primária em saúde do município.

Os gastos com tratamentos referentes ao câncer de pulmão nos EUA são estimados em 14,73 bilhões de dólares no ano de 2020 (Mariotto et al., 2011). Além disso, o impacto econômico do câncer de pulmão na Europa é substancial, com custos diretos do cuidado de pacientes com a doença totalizando mais de $€ 3$ bilhões por ano (ERS, 2013).

Ademais, conforme o DATASUS, as despesas relacionadas às neoplasias malignas de pulmão, traqueia e brônquios atingiram cerca de R\$ 37.5 milhões em 2017, R\$ 38.1 milhões em 2018 e R\$ 41.6 milhões em 2019 e R\$ 35.2 milhões em 2020 no Brasil. Assim, observa-se consonância entre o cenário nacional e o da cidade de Altamira, pois ocorreu queda expressiva dos custos hospitalares com os cânceres supracitados nos dois contextos no último ano. Essa diminuição pode estar associada à redução nacional da busca por atendimento hospitalar por parte dos pacientes oncológicos em decorrência do temor causado pela pandemia causada pelo SARS-CoV-2 (Araujo et al., 2021).

A presente pesquisa identificou cerca de $\mathrm{R} \$ 29.682,22$ como gastos totais relacionados à cidade de Altamira em decorrência das doenças neoplásicas estudas. Desse valor, apenas $\mathrm{R}$ \$ 4.297,74 foram gastos com atendimentos hospitalares no município para pacientes com câncer de pulmão, brônquios e traqueia (Tabela 2). Essas despesas são bastante inferiores em comparação às encontradas para DPOC, que foi superior a R \$ 144 mil. Isso decorre provavelmente do fato de Altamira não possuir uma rede de alta complexidade em oncologia. Dessa forma, os pacientes diagnosticados com neoplasias precisam deslocar-se para outros centros de referência no estado do Pará, o que pode dificultar o diagnóstico precoce da doença e, assim, reduzir a sobrevida do doente.

O atraso no diagnóstico da neoplasia maligna de pulmão, brônquios e traqueia constitui um dos principais desafios enfrentados no Brasil. Ademais, as características evolutivas da neoplasia pulmonar, fatores relacionados ao sistema de saúde e ao próprio paciente podem ser responsabilizados por tal diagnóstico tardio. É importante destacar que o acesso a exames de imagem é limitado em várias regiões brasileiras, principalmente nas regiões norte e nordeste, logo pacientes que residem nesses locais sofrem impactos maiores com a falta de um rastreamento efetivo (Araujo et al., 2018). Desse modo, para Barros et al., (2006), medidas de detecção precoce tornam-se importantes, visto que a ressecção cirúrgica, única abordagem terapêutica que oferece cura potencial, é mais efetiva em estágios clínicos iniciais. 
Ao mencionar os impactos da DPOC para o Sistema Único de Saúde (SUS), conforme o Protocolo Clínico e Diretrizes Terapêuticas da Doença Pulmonar Obstrutiva Crônica do Ministério da Saúde, têm-se que essa patologia foi a quinta maior causa de internação nos últimos 10 anos (Brasil, 2013). Para Malta et al., (2017), a epidemia das doenças crônicas não transmissíveis resulta em problemas devastadores para indivíduos, famílias e comunidades, além de sobrecarregar os sistemas de saúde. Segundo Marques et al., (2014), os custos com a DPOC poderiam ser reduzidos já que essa patologia é classificada internacionalmente como Ambulatory Care Sensitive Condition (ACSCs), ou seja, passíveis de serem evitadas com maiores investimentos na Atenção Primária.

Os desafios para prevenção da DPOC e neoplasia maligna das vias aéreas inferiores estão relacionados principalmente à exposição à fumaça do tabaco (Rabahi, 2013). Na atenção primária, o Programa Nacional de Controle do Tabagismo (PNCT) é a diretriz do SUS para a diminuição da prevalência de fumantes (Portes et al., 2014). Para queda do número de casos dessas doenças, portanto, é necessário a capacitação da equipe multidisciplinar nas Unidades de Saúde da Família acerca do Protocolo Clínico e Diretrizes Terapêuticas do Tabagismo (Brasil, 2020). Dessa forma, mais tabagistas poderão, com o devido apoio de terapias medicamentosas ou não, abandonar tal vício mortal e, logo, evitar graves patologias respiratórias.

A saúde de trabalhador também deve ser prioridade para o governo, tendo em vista que é determinante para o combate às patologias respiratórias abordadas neste estudo, pois a exposição ocupacional é fator evitável ou no mínimo capaz de ser mitigado. Assim, ocupações perigosas para a integridade pulmonar, como mineração, exploração de madeira e garimpagem, exigem carga de trabalho reduzida e atenuação na medida do possível da exposição às substâncias tóxicas para a saúde pulmonar humana, para prevenir tanto a DPOC quanto o câncer de pulmão, traqueia e brônquios (Gelati et al., 2017; Vinnikov et al., 2019). É válido ressaltar a importância de empregadores oferecerem Equipamentos de Proteção Individual (EPIs) e capacitação para a utilização adequada desses, porquanto, caso seu uso seja inadequado, tais itens apenas contribuirão para o desconforto do usuário e o para o perigo de exposição a agentes maléficos à respiração (Bagatin \& Kitamura, 2006).

Além disso, as autoridades estatais precisam urgentemente oferecer para a população altamirense acesso gratuito e facilitado a exames para avaliação pulmonar requeridos por profissionais de saúde, como espirometria, tomografia computadorizada e ressonância magnética, com o objetivo de possibilitar o diagnóstico precoce das patologias tratadas neste trabalho e, assim, de melhorar o prognóstico do doente.

Portanto, o enfisema pulmonar, a bronquite crônica e as neoplasias malignas do trato respiratório inferior provocam grande sofrimento físico e emocional a quem acomete, além de custos financeiros significativos para o SUS no Brasil. Além disso, é válido destacar que indivíduos do sexo masculino, de idade avançada e de etnia parda são as mais frequentes vítimas que necessitaram de internação hospitalar em decorrência das doenças abordadas neste estudo em Altamira, no Pará. Logo, é imprescindível o fortalecimento de medidas de prevenção e de rastreio contra essas enfermidades, com o objetivo de mitigar a exposição da população altamirense a fatores de risco, dentre os quais se destaca o tabagismo e a exposição ocupacional.

\section{Conclusão}

Os resultados deste estudo ímpar destacam os gastos públicos hospitalares com DPOC e neoplasias malignas do trato respiratório inferior em Altamira, no estado do Pará. A morte precoce, a redução de anos de vida com saúde plena, o sofrimento emocional do paciente e de sua família e os significativos custos financeiros evitáveis são efeitos dessas doenças. Dessa forma, o diagnóstico precoce, práticas de educação em saúde e de apoio assistencial para cessação do tabagismo e para a proteção da saúde do trabalhador são ferramentas fundamentais a fim de obter redução das repercussões econômicas dessas enfermidades para o sistema público de saúde brasileiro e melhoria da qualidade de vida da população altamirense. 


\section{Referências}

Algranti, E., Buschinelli, J. T. P., \& De Capitani, E. M. (2010). Câncer de pulmão ocupacional. Jornal Brasileiro de Pneumologia, 36(6), 784-794.

Araujo, L. H., Baldotto, C., Castro Jr, G. d., Katz, A., Ferreira, C. G., Mathias, C., \& Tabacof, J. (2018). Câncer de pulmão no Brasil. Jornal Brasileiro de Pneumologia, 44(1), 55-64.

Araujo, S. E. A., Leal, A., Centrone, A. F. Y., Teich, V. D., Malheiro, D. T., Cypriano, A. S., \& Klajner, S. (2021). Impacto da COVID-19 sobre o atendimento de pacientes oncológicos: experiência de um centro oncológico localizado em um epicentro Latino-Americano da pandemia. Jornal Einstein, 19, eAO6282.

Bagatin, E., \& Kitamura, S. (2006). História ocupacional. Jornal brasileiro de pneumologia, 32, S12-S16.

Bagatin, E., Jardim, J. R. B., \& Stirbulov, R. (2006). Doença pulmonar obstrutiva crônica ocupacional. Jornal Brasileiro de Pneumologia, 32, S35-S40.

Balbani, A. P. S., \& Montovani, J. C. (2005). Métodos para abandono do tabagismo e tratamento da dependência da nicotina. Revista Brasileira de Otorrinolaringologia,71(6), 820-827.

Barcenas, C. H., Delclos, G. L., El-Zein, R., Tortolero-Luna, G., Whitehead, L. W., \& Spitz, M. R. (2005). Wood dust exposure and the association with lung cancer risk. American journal of industrial medicine, 47(4), 349-357. https://doi.org/10.1002/ajim.20137.

Barnes, P. J. (2004). Alveolar macrophages as orchestrators of COPD. COPD: Journal of Chronic Obstructive Pulmonary Disease, 1(1), 59-70.

Barros, J. A., Valladares, G., Faria, A. R., Fugita, E. M., Ruiz, A. P., Vianna, A. G. D., \& Oliveira, F. A. M. (2006). Diagnóstico precoce do câncer de pulmão: o grande desafio. Variáveis epidemiológicas e clínicas, estadiamento e tratamento. Jornal Brasileiro de Pneumologia, $32(3), 221-227$.

Bolund, A. C., Miller, M. R., Jacobsen, G. H., Sigsgaard, T., \& Schlünssen, V. (2018). New-onset COPD and decline in lung function among wood dustexposed workers: re-analysis of a 6-year follow-up study. Annals of work exposures and health, 62(9), 1064-1076.

Brasil, M. d. S. (2013). Ministério da Saúde. Doença pulmonar obstrutiva crônica. Secretaria de Atenção à Saúde. $203-232$.

Brasil, M. d. S. (2013). Ministério da Saúde. Portaria nº 609, de 06 de junho de 2013. Protocolo Clínico e Diretrizes Terapêuticas - Doença pulmonar obstrutiva crônica.

Brasil, M. d. S. (2020). Ministério da Saúde. Secretaria de Ciência, Tecnologia, Inovação e Insumos Estratégicos em Saúde - SCTIIE. Departamento de Gestão e Incorporação de Tecnologias e Inovações em Saúde - DGITIS. Coordenação de Gestão de Protocolos Clínicos e Diretrizes Terapêuticas - CPCDT, 1 -61 .

Brasil, M. d. S. (2020). Tipos de câncer: câncer de pulmão; estatísticas 2020. Ministério da Saúde.

Brasil. Ministério da Saúde. (2006). Portaria n ${ }^{\circ}$ 1.097, de 22 de maio de 2006. Define o processo de Programação Pactuada e Integrada da Assistência em Saúde seja um processo instituído no Sistema Único de Saúde.

Burns, D. M. (2003). Tobacco-related diseases. Paper presented at the Seminars in oncology nursing. Elsevier, 4, $244-249$.

Carmo, C. B. d. S., Silva, R. D., \& Teixeira, R. d. C. (2014). Perfil epidemiológico de pacientes com câncer de pulmão em um hospital público de referência oncológica do estado do Pará. Revista Paraense de Medicina,. 28.

Cruz, M. M., \& Pereira, M. (2020). Epidemiology of Chronic Obstructive Pulmonary Disease in Brazil: a systematic review and meta-analysis. Jornal Ciência Saúde Coletiva, 25, 4547-4557.

DATASUS. (2021). Cadastro Nacional de Estabelecimentos de Saúde - CNES. http://cnes.datasus.gov.br/pages/estabelecimentos/consulta.jsp

DATASUS. (2021). Sistema de Informações Hospitalares - SIH. http://tabnet.datasus.gov.br/cgi/deftohtm.exe?sih/cnv/nipa.def

DATASUS. (2021). Sistema de Informações Hospitalares - SIH. http://tabnet.datasus.gov.br/cgi/deftohtm.exe?sih/cnv/nrpa.def

de Groot, P. M., Wu, C. C., Carter, B. W., \& Munden, R. F. (2018). The epidemiology of lung cancer. Translational lung cancer research, 7(3), $220-233$.

Doll, R., \& Hill, A. B. (1950). Smoking and carcinoma of the lung. British medical journal., 2(4682), 739-748.

Ejike, C. O., Dransfield, M. T., Hansel, N. N., Putcha, N., Raju, S., Martinez, C. H., \& Han, M. K. (2019). Chronic obstructive pulmonary disease in America's black population. American journal of respiratory critical care medicine, 200(4), 423-430.

ERS. (2013). The economic burden of lung disease. European Lung White Book. Sheffield: European Respiratory Society (ERS), $16-27$.

Fagan, P., Moolchan, E. T., Lawrence, D., Fernander, A., \& Ponder, P. K. (2007). Identifying health disparities across the tobacco continuum. Addiction journal, 102, 5-29.

Fainguelernt, M. B. (2016). A trajetória histórica do processo de licenciamento ambiental da usina hidrelétrica de Belo Monte. Ambiente \& Sociedade, 19(2), $245-264$.

Fainguelernt, M. B. (2020). Impactos da Usina Hidrelétrica de Belo Monte: uma análise da visão das populações ribeirinhas das reservas ex trativistas da Terra do Meio. Civitas-Revista de Ciências Sociais, 20(1), 43-52. 
Ferlay, J., Colombet, M., Soerjomataram, I., Mathers, C., Parkin, D. M., Piñeros, M., \& Bray, F. (2019). Estimating the global cancer incidence and mortality in 2018: GLOBOCAN sources and methods. 144(8), 1941-1953.

Forey, B. A., Thornton, A. J., \& Lee, P. N. (2011). Systematic review with meta-analysis of the epidemiological evidence relating smoking to COPD, chronic bronchitis and emphysema. BMC pulmonary medicine journal, 11(1), 1-61.

Freire, L. M., de Lima, J. S., \& da Silva, E. V. (2018). Belo Monte: fatos e impactos envolvidos na implantação da usina hidrelétrica na região Amazônica Paraense. Sociedade \& Natureza, 30(3), 18-41.

Gelati, T. R., Cezar-Vaz, M. R., Bonow, C. A., Couto, A. M. D., Costa, V. Z. D., \& Oliveira, A. M. N. D. (2017). Alterações respiratórias em trabalhadores: estudo de portuários avulsos. Revista Gaúcha de Enfermagem, 38(4).

Girdler-Brown, B. V., White, N. W., Ehrlich, R. I., \& Churchyard, G. J. (2008). The burden of silicosis, pulmonary tuberculosis and COPD among former Basotho goldminers. American journal of industrial medicine, 51(9), 640-647.

GOLD. (2020). Global strategy for the diagnosis, management, and prevention of chronic obstructive pulmonary disease. Global Initiative for Obstructive Lung Disease (GOLD). 1, 80-81.

Guarascio, A. J., Ray, S. M., Finch, C. K., \& Self, T. H. (2013). The clinical and economic burden of chronic obstructive pulmonary disease in the USA. ClinicoEconomics outcomes research: CEOR, 5, 235-245.

Hammond, E. C., \& Horn, D. (1958). Smoking and death rates: Report of forty-four months of follow-up of 187 - 783 men. II. Death rates by cause. . Journal of the American Medical Association (JAMA), 166, 1294-1308.

Hancock, D. G., Langley, M. E., Chia, K. L., Woodman, R. J., \& Shanahan, E. M. (2015). Wood dust exposure and lung cancer risk: a meta-analysis. Occupational and environmental medicine, 72(12), 889-898.

Hochhegger, B., Alves, G. R. T., Irion, K. L., Fritscher, C. C., Fritscher, L. G., Concatto, N. H., \& Marchiori, E. (2015). PET/TC em câncer de pulmão: indicações e achados. JJornal Brasileiro de Pneumologia, 41(3), 264-274.

Howlader, N., Noone, A. M., Krapcho, M., Miller, D., Brest, A., Yu, M., \& Cronin, K. A. (2016). SEER Cancer Statistics Review, 1975-2016, National Cancer Institute. Bethesda, MD. https://seer.cancer.gov/csr/1975_2016/

IBGE. (2010). Instituto Brasileiro de Geografia e Estatística. Densidade demográfica. https://cidades.ibge.gov.br/brasil/pa/altamira/panorama

IBGE. (2018). Instituto Brasileiro de Geografia e Estatística. Trabalho e Rendimento. https://cidades.ibge.gov.br/brasil/pa/altamira/panorama

IBGE. (2020). Instituto Brasileiro de Geografia e Estatística. Área Territorial. https://www.ibge.gov.br/cidades-e-estados/pa/altamira.html

IBGE. (2020). Instituto Brasileiro de Geografia e Estatística. População estimada. https://cidades.ibge.gov.br/brasil/pa/altamira/panorama

INCA. (2011). Instituto Nacional de Câncer. Pesquisa Especial de Tabagismo (PETab). Relatório Brasil.: Ministério da Saúde, Instituto Nacional de Câncer/Organização Pan-Americana da Saúde. 26-36.

INCA. (2020). Instituto Nacional de Câncer. Estatísticas de câncer. Incidência estimada conforme a localização primária do tumor e sexo. https://www.inca.gov.br/numeros-de-cancer

Jezler, S., Holanda, M. A., Jose, A., \& Franca, S. (2007). Ventilação mecânica na doença pulmonar obstrutiva crônica (DPOC) descompensada. Jornal Brasileiro de Pneumologia, 33, 111-118.

Ladislau, J. L. D. B., Póvoa, M. M., Sucupira, I. M. C., Galardo, A. K. R., Damasceno, O. C., Cardoso, B. D. S., \& Tauil, P. L. (2016). Malaria control in area of hydroelectric construction on the Amazonian ecosystem can succeed?

Malta, D. C., Bernal, R. T. I., Lima, M. G., Araújo, S. S. C. d., Silva, M. M. A. d., Freitas, M. I. d. F., \& Barros, M. B. d. A. (2017). Doenças crônicas não transmissíveis e a utilização de serviços de saúde: análise da Pesquisa Nacional de Saúde no Brasil. Revista de Saúde Pública, 51, 1s - 4s.

Malta, D. C., Vieira, M. L., Szwarcwald, C. L., Caixeta, R., Brito, S. M. F., \& Reis, A. A. C. d. (2015). Tendência de fumantes na população Brasileira segundo a Pesquisa Nacional de Amostra de Domicílios 2008 e a Pesquisa Nacional de Saúde 2013. Revista Brasileira de Epidemiologia, 18, 45-56.

Mariotto, A. B., Robin Yabroff, K., Shao, Y., Feuer, E. J., \& Brown, M. L. (2011). Projections of the cost of cancer care in the United States: 2010-2020. Journal of the National Cancer Institute, 103(2), 117-128.

Marques, A. P., Montilla, D. E. R., Almeida, W. d. S. d., \& Andrade, C. L. T. d. (2014). Internação de idosos por condições sensíveis à atenção primária à saúde. Revista de Saúde Pública, 48, 817-822.

MMA. (2013). Ministério do Meio Ambiente. Serviço florestal brasileiro. Edital de licitação para concessão florestal, Concorrência nº 03/2013 - Anexo 3. Contextualização geográfica, social e ambiental da floresta nacional de Altamira e entorno, 28.

Moran-Mendoza, O., Pérez-Padilla, J. R., Salazar-Flores, M., \& Vazquez-Alfaro, F. (2008). Wood smoke-associated lung disease: a clinical, functional, radiological and pathological description. The international journal of tuberculosis and lung disease: the official journal of the International Union against Tuberculosis and Lung Disease, 12(9), 1092-1098.

Neto, J. Q. D. M., \& Herrera, J. A. (2016). Altamira-PA: novos papéis de centralidade e reestruturação urbana a partir da instalação da UHE Belo Monte. Confins. Revue franco-brésilienne de géographie/Revista franco-brasilera de geografia, (28).

Peng, C., Yan, Y., Li, Z., Jiang, Y., \& Cai, Y. (2020). Chronic obstructive pulmonary disease caused by inhalation of dust: A meta-analysis. Medicine, 99(34). 
Pinto M, Bardach A, Palacios A, Biz AN, Alcaraz A, Rodríguez B, \& Pichon-Riviere, A. (2017). Carga de doença atribuível ao uso do tabaco no brasil e potencial impacto do aumento de preços por meio de impostos. Documento técnico IECS N ${ }^{\circ} 21$. Instituto de Efectividad Clínica y Sanitaria, Buenos Aires, Argentina, 32.

Pinto, M. T., Pichon-Riviere, A., \& Bardach, A. (2015). Estimativa da carga do tabagismo no Brasil: mortalidade, morbidade e custos. Cadernos de Saúde Pública, 31, 1283-1297.

Poinen-Rughooputh, S., Rughooputh, M. S., Guo, Y., Rong, Y., \& Chen, W. (2016). Occupational exposure to silica dust and risk of lung cancer: an updated meta-analysis of epidemiological studies. BMC public health, 16(1), 1-17.

Portes, L. H., Machado, C. V., Turci, S. R. B., Figueiredo, V. C., Cavalcante, T. M., \& Silva, V. L. D. C. (2018). A Política de Controle do Tabaco no Brasil: um balanço de 30 anos. Ciência \& Saúde Coletiva, 23, 1837-1848.

Portes, L. H., Campos, E. M. S., Teixeira, M. T. B., Caetano, R., \& Ribeiro, L. C. (2014). Ações voltadas para o tabagismo: análise de sua implementação na Atenção Primária à Saúde. Ciência \& Saúde Coletiva, 19, 439-448.

Rabahi, M. F. (2013). Epidemiologia da DPOC: enfrentando desafios. Pulmão RJ, 22 4-8.

Romão, E., Pontes, A., Gutjahr, A. L., \& Torres, W. R. (2017). Análise temporal do uso e da cobertura do solo nas áreas desflorestadas do município de Altamira, Pará. Enciclopédia Biosfera, 14(25).

Sales, B. R. e. a. (2018). Perfil Epidemiológico dos pacientes com DPOC no município de Caratinga. Brazilian Journal of Surgery and Clinical Research, 23(1), $17-21$.

SBPT. (2004). II Consenso Brasileiro de Doença Pulmonar Obstrutiva Crônica (DPOC). Sociedade Brasileira de Pneumologia e Tisiologia (SBPT). Jornal Brasileiro de Pneumologia, 30(5), 1-42.

SESPA. (2016). Secretaria de Estado de Saúde Pública do Pará. Plano Estadual de Saúde (PES) 2016 - 2019. Governo do estado do Pará, 98.

Silva, C. N. D., Rodrigues, J. E. C., Luz, L. M. D., \& Ponte, F. C. D. (2013). Atlas geográfico escolar do Estado do Pará.

Soares, S., Costa, I., Neves, A. L., \& Couto, L. (2010). Caracterização de uma população com risco acrescido de DPOC. Revista Portuguesa de Pneumologia, $16(2), 237-252$.

Society, A. C. (2010). The Global Economic Cost Of Cancer. Distribution Of Economic Impact. In: American Cancer Society. American Cancer Society, [S. 1.], 14 .

Thun, M. J., Day-Lally, C., Myers, D. G., Calle, E. E., Flanders, W., Zhu, B., Heath, C. (1982). Trends in tobacco smoking and mortality from cigarette use in Cancer Prevention Studies I (1959 through 1965) and II (1982 through 1988). Changes in Cigarette-Related Disease Risks and Their Implication for Prevention and Control: Smoking and Tobacco Control Monograph 8. Bethesda, Md: US Deptof Health and Human Services, Public Health Service, National Institutes of Health, National Cancer Institute, cap. 4, 305-382.

Torres, K. D. P., Cunha, G. M., \& Valente, J. G. (2018). Tendências de mortalidade por doença pulmonar obstrutiva crônica no Rio de Janeiro e em Porto Alegre, 1980-2014. Revista Epidemiologia e Serviços de Saúde, 27, e2017139.

Varmaghani, M., Dehghani, M., Heidari, E., Sharifi, F., Moghaddam, S. S., \& Farzadfar, F. (2019). Global prevalence of chronic obstructive pulmonary disease: systematic review and meta-analysis. East Mediterr Health Journal, 25(1), 47-57.

Velásquez, C., Villas Boas, A., \& Schwartzman, S. (2006). Desafio para a gestão ambiental integrada em território de fronteira agrícola no oeste do Pará. Revista de Administração pública, 40(6), 1061-1075.

Veríssimo, G., Mendonça, R., \& Meyer, A. (2013). Mortalidade de mineiros brasileiros por câncer entre 1979-2005. Cadernos Saúde Coletiva, 21(3), 281-288.

Vinnikov, D., Raushanova, A., Kyzayeva, A., Romanova, Z., Tulekov, Z., Kenessary, D., \& Auyezova, A. (2019). Lifetime occupational history, respiratory symptoms and chronic obstructive pulmonary disease: results from a population-based study. International journal of chronic obstructive pulmonary disease, 14,3025 .

WHO. (2020). World Health Organization.Top 10 global causes of disability-adjusted life years (DALYs). Global Health Estimates: Life expectancy and leading causes of death and disability. https://www.who.int/data/gho/data/themes/mortality-and-global-health-estimates

Zamboni, M. (2002). Epidemiologia do câncer do pulmão. Jornal Brasileiro de Pneumologia, 28(1), 41-47. 